\title{
High Performance of Linear Permanent Magnet Synchronous Motor Drive using Unscented Kalman Filter
}

\author{
B. Arundhati ${ }^{1}$, S. Koteswar Rao ${ }^{2}$ and Tai-hoon $\mathrm{Kim}^{3 *}$ \\ ${ }^{1}$ Electrical \& Electronics Engineering Department, Vignan's Institute of \\ Information Technology, Visakhapatnam-530049, India \\ ${ }^{2}$ Electronics and Communications Engineering Department, \\ KL University, Guntur-522502, India \\ ${ }^{3}$ Department of Convergence Security, Sungshin Women's University, 249-1, \\ Dongseon-dong 3-ga, Seoul 136-742, South Korea \\ ${ }^{1}$ b_arundhati@rediffmail.com, 2 rao.sk9@gmail.com, $3 *$ taihoonn@daum.net
}

\begin{abstract}
Precise operation of Permanent Magnet Linear Synchronous Motor (LPMSM) requires information of speed and position of the motor which is generally sensed by a linear encoder. The cost of linear encoder is about one third of the motor. Installation and maintenance of linear encoders are problematic in many cases. To overcome this problem and to reduce the cost of drive, sensor less control of LPMSM drive is proposed in this work. Different estimation methods are in vogue for determination of the mover position and speed from the knowledge of the motor current and voltage. Extended Kalman Filter $(E K F)$ is one amongst them. In this method, the system matrix is linearised up to first order Taylor expansion, and this causes errors owing to truncation of higher order terms. To minimize the estimation error, Unscented Kalman Filter (UKF) is implemented in this paper. Observations confirm superior performance of UKF over EKF in tracking the speed during transient conditions of sudden changes in load and speed. Simulation is performed extensively using Simulink / embedded MATLAB and is validated experimentally using DS1104 controller board.
\end{abstract}

Keywords: Linear Synchronous Motor, Nonlinear estimation, Extended and Unscented Kalman Filter \& DS1104 controller board

\section{Introduction}

Linear Permanent Magnet Synchronous Motor (LPMSM) is a special case of linear motors. It competes well with the others in its field in view of the applications desirous of accurate and precise control. Linear motors continue to remain front runners in the area of advanced motoring applications. Their utility makes them a subject of intense research for improvements in control and performance. Linear Synchronous Motors tend have an edge over Linear Induction Motors in view of their high precision and energy density [1]. Input information of speed and position are necessary for precise control of LPMSM which is generally achieved by linear encoders. Linear encoders have certain drawbacks as those of cost and space requirements. They tend to be the weakest link of the system in aggressive conditions. These disadvantages not only reduce the reliability of the system but also degrade the drive's performance. The answer to this lies in the possible replacement of the linear encoder by an estimator to estimate the position and speed of the motor.

Extensive research has been carried out in recent years to estimate the position and speed of the motor. Estimation methods as those of Sliding mode observer, Luenberger observer,

Received (July 19, 2017), Review Result (September 18, 2017), Accepted (November 6, 2017)

* Corresponding Author 
Neuro-Fuzzy observer and EKF etc. have been in vogue. For nonlinear systems sliding mode observer is very much suitable and shows robust performance with parameter variation and load variation [2]. On the flip side it uses high speed switching to converge to the sliding mode plane. Because of discontinuous switching, it fails to give accurate results at low speed operation of the motor. MRAS is simple in structure and easier to execute, hence more popular [3]. However, the accuracy of reference model output mainly influences the precision of speed estimation [4]. EKF is a nonlinear stochastic estimator which is extensively used for state estimation [5]. The last few years have witnessed a profound use of EKF for nonlinear estimation. Few inherent demerits associated with the estimation process make EKF an unfavorable choice. In EKF, the nonlinear system matrix is linearised to the 1st order expansion of Taylor's series to minimize the computational complexity [6]. Since during linearization of the state matrix higher order terms are truncated, as a result large estimation errors occur in case of EKF [7]. Calculation of Jacobians matrix and linearization of system matrix are some of the flaws associated with EKF. To overcome these drawbacks, UKF is proposed, which does away with linearization of the system matrix [8].

\section{Modeling of LPMSM}

The dynamic modeling equations of LPMSM [9]

$$
\begin{aligned}
& U_{q}=r_{s} I_{q}+l_{q} \frac{d i_{q}}{d t}+\frac{\pi}{\tau} l_{d} v_{r} I_{d}+\frac{\pi}{\tau} v_{r} \Psi_{f} \\
& U_{d}=r_{s} I+l_{d} \frac{d i_{d}}{d t}-\frac{\pi}{\tau} v_{r} l_{q} I_{q}
\end{aligned}
$$

Where the motor voltages in d-q axis are $U_{d} \& U_{q}$, currents are $I_{d} \& I_{q}$, effective armature inductances are $l_{d} \& l_{q} . r_{s}$ is the per phase resistance, $\Psi_{f}$ is the permanent magnet flux linkage per phase. The machine dynamism relates electromagnetic thrust $F_{e}$, mass of the mover $M$, frictional coefficient $B$, linear speed $v_{r}$ and load thrust $F_{l}$ is as below:

$$
F_{e}=M \frac{d v_{r}}{d t}+B v_{r}+F_{l}
$$

The electromagnetic thrust for the surface mounted LPMSM is:

$F_{e}=\frac{3}{2} \frac{\pi}{\tau} \Psi_{f} I_{q}=K_{f} I_{q}$

Where $K_{\mathrm{f}}$ is the thrust constant in N/A.

\section{Nonlinear Estimation Techniques}

\section{A. Extended Kalman Filter}

EKF is an optimum state-observer which is used to determine the states of a non-linear system affected by measurement noise and modeling inaccuracies. In this paper using EKF the LPMSM mover position and speed is estimated.

The system equations are linearised by partial derivative and discretized using Taylor series. The discretized and linearised system matrix G', Input matrix H' \& Output matrix C' are 
$G^{\prime}=\left[\begin{array}{cccc}1-\frac{R_{S}}{L_{d}} T & v_{r} \frac{\pi}{\tau} \frac{L_{q}}{L_{d}} T & i_{q} \frac{\pi}{\tau} \frac{L_{q}}{L_{d}} T & 0 \\ -v_{r} \frac{\pi}{\tau} \frac{L_{d}}{L_{q}} T & 1-\frac{R_{s}}{L_{q}} T & -\frac{\pi}{\tau}\left[\frac{\Psi_{p m}}{L_{q}}+\frac{L_{d}}{L_{q}} i_{q}\right] T & 0 \\ 0 & \frac{K_{f}}{M} T & 1-\frac{B}{M} T & 0 \\ 0 & 0 & T & 0\end{array}\right]$
$H^{\prime}=\left[\begin{array}{cc}\frac{1}{L_{d}} T & 0 \\ 0 & \frac{1}{L_{q}} T \\ 0 & 0 \\ 0 & 0\end{array}\right]$ and $\mathrm{C}^{\prime}=\left[\begin{array}{llll}T & 0 & 0 & 0 \\ 0 & T & 0 & 0\end{array}\right]$

\section{B. Unscented Kalman Filter}

$\mathrm{UKF}$ is a recursive type, derivative free nonlinear state estimator [10]. UKF works on principle of Unscented Transformation (UT). UKF uses deterministic sampling approach to overcome the flaws of EKF algorithm, in this UKF algorithm state distribution is approximated using sigma points (a set of vectors) from the knowledge of the covariance and mean of the state vectors, states of the system can be estimated accurately with the help of these sigma points [11]. The Figure 1 depicts the estimation approach of both the estimators.
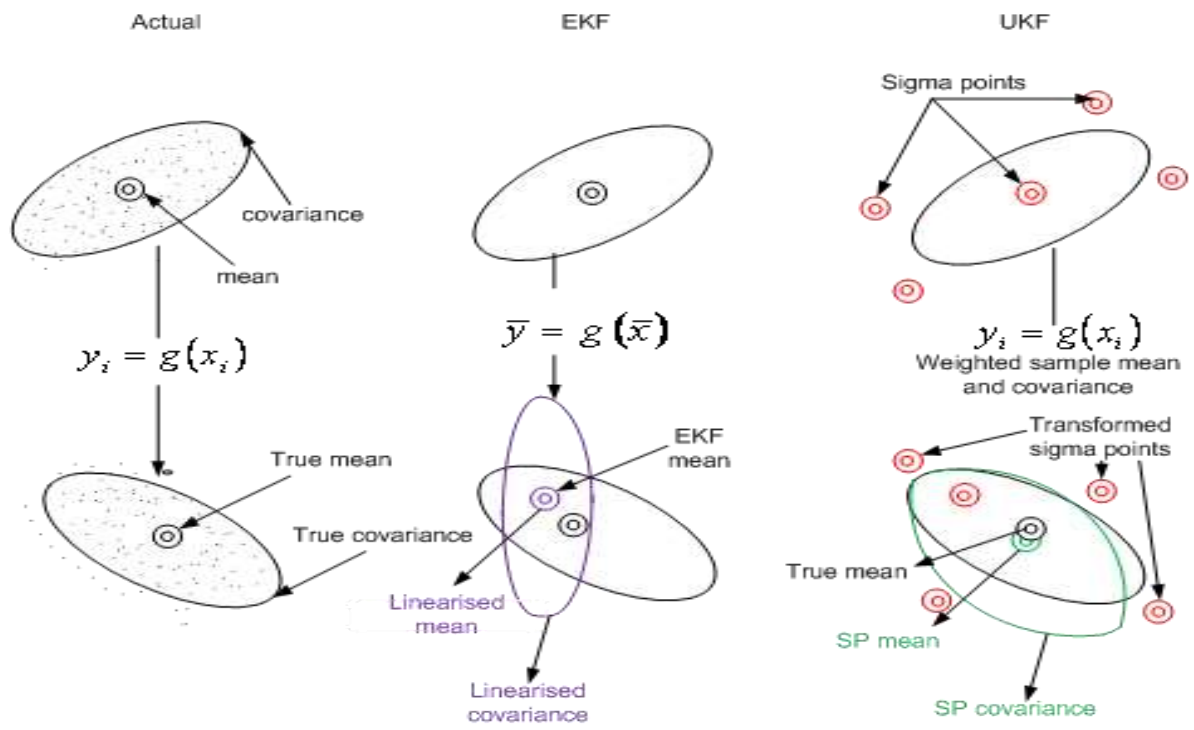

Figure 1. Comparison between EKF and UKF Estimation Approach

The three basic steps in the UKF algorithm comprise of Prediction of the states, weighting and correction of the states. Sigma point and hence sigma vectors are computed in weighting step. In UKF sigma points are calculated deterministically from the available covariance and mean. In the next step, those points are transformed with nonlinear function to estimate the transformed covariance and mean. Suppose $K$ no. state variables represented as $x$ has covariance $\mathrm{P}$ and mean $\bar{x}$, matrix $\chi$ of $2 K+1$ sigma vectors $x_{i}$ (with related weights $w_{i}$ ), are represented as follow.

$$
x_{i}=(\sqrt{(K+\zeta) P})_{i}^{T} \pm \bar{x} \quad i=1, \ldots \ldots \ldots, K
$$

The weighting coefficients are given as 


$$
\begin{gathered}
w_{0}^{(c)}=\zeta /(K+\zeta) \\
w_{0}^{(m)}=\left(1-a^{2}+b\right)+\zeta /(K+\zeta) \\
w_{i}^{(c)}=\zeta / 2(K+\zeta)=w_{i}^{(n)} \quad i=1, \ldots \ldots \ldots, 2 K
\end{gathered}
$$

Where $\zeta=K\left(a^{2}-1\right)$ is a scaling parameter. And $\alpha$ decides extend of the sigma points in the region of $\bar{x}$ and its value is about 0.001 , distribution of $x$ is determined by $b$ and its value is considered as 2 for Gaussian distributions. These sigma vectors are distributed around the nonlinear function [12].

$$
y_{i}=g\left(x_{i}\right) \quad i=0,1, \ldots \ldots, 2 K
$$

$w_{0}^{(c)}$ and $w_{o}^{(m)}$ are the weights of initialized target covariance matrix and state vector respectively, $w_{i}^{(c)}$ and $w_{i}^{(m)}$ are the weights of target state sigma point vector and their covariance matrix.

The LPMSM represented in discrete state space form with noise as:

$$
\begin{aligned}
& x_{n+1}=f\left(x_{n}, u_{n}\right)+\mathcal{W}_{k} \\
& y_{n}=\mathcal{G}\left(x_{n}\right)+\mathcal{V}_{k}
\end{aligned}
$$

The proposed UKF algorithm steps are as follows:

1. State vectors calculation with $2 \mathrm{~K}+1$ sigma points given as

$$
\chi_{n-1}=\left[x_{n-1} ; \quad x_{n-1} \pm(\sqrt{(K+\zeta) P})_{i}^{T}\right] i=1, \ldots \ldots, K
$$

2. Transformation of sigma points

$$
x_{i, n \mid n-1}=f\left(x_{i, n-1}, u_{n}\right)
$$

3. The predicted state is as given

$$
x_{n \mid n-1}=\sum_{i=0}^{2 k} w_{i}^{(c)} *\left(x_{i, n \mid n-1} x_{i}\right)
$$

4. Covariance is Predicted from initial conditions is given as

$$
\mathcal{P}_{n \mid n-1}=\sum_{i=0}^{2 k} w_{i}^{(m)} *\left[x_{i, n \mid n-1}-x_{n \mid n-1}\right]\left[x_{i, n \mid n-1}-x_{n \mid n-1}\right]^{T}+Q_{n}
$$

5. Sigma points time update

$$
\chi_{n \mid n-1}=\left[\begin{array}{cc}
x_{n \mid n-1} & x_{n \mid n-1} \pm\left(\sqrt{(K+\zeta) \mathcal{P}_{n \mid n-1}}\right)_{i}^{T}
\end{array}\right] i=
$$

6. Sigma points transformation through output equation.

$$
y_{i, n \mid n-1}=g\left[x_{i, n \mid n-1}\right]
$$

7. Measurement Prediction is given as

$$
y_{n \mid n-1}=\sum_{i=0}^{2 k} w_{i}^{(c)} * y_{i, n \mid n-1}
$$

8. The covariance of output is updated as

$$
\mathcal{P}_{y y}=\sum_{i=0}^{2 k} w_{i}^{(m)}\left[y_{i, n \mid n-1}-y_{n \mid n-1}\right]\left[y_{i, n \mid n-1}-y_{n \mid n-1}\right]^{T}+\mathcal{R}_{n}
$$

9. The updated cross covariance

$$
\mathcal{P}_{x y}=\sum_{i=0}^{2 k} w_{i}^{(m)} *\left[x_{i, n \mid n-1}-x_{n \mid n-1}\right]\left[y_{i, n \mid n-1}-y_{n \mid n-1}\right]^{T}
$$


10. Kalman gain is premeditated as

$$
K n=\mathcal{P}_{x y} * \mathcal{P}_{y y}^{-1}
$$

11. The Predicted measurement update

$$
x_{n \mid n}=K_{n} *\left[y_{n}-y_{n \mid n-1}\right]+x_{n \mid n-1}
$$

12. Error covariance update is given as

$$
P_{n \mid n}=\mathcal{P}_{n \mid n-1}-K_{n} \mathcal{P}_{y y} * K_{n}^{T}
$$

\section{Proposed Controller with Nonlinear Estimator}

The proposed control scheme including the estimator is as shown in Figure 1. The outer speed loop consists of a PI controller to which 'error in speed' is input to get 'reference thrust' as the output. The reference current in $\mathrm{d}$ and $\mathrm{q}$ axis is obtained using field oriented Control, setting direct axis current as zero and generates only q-axis reference current. The inner current loop consists of nonlinear controller and gives control voltage; the motor position and speed is estimated from the inputs of motor current and voltage using EKF \& UKF algorithm. These estimates are compared with actual values.

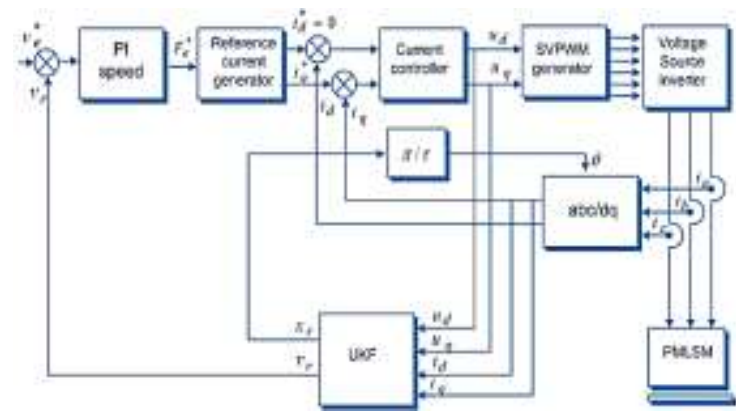

Figure 2. Schematic Diagram of Proposed LPMSM Drive

\section{Result Analysis \& Discussion}

The performance of UKF over EKF is depicted in various case studies with the help of MATLAB simulation. Finally, the elegance of estimation comparison has been reproduced in the form of r.m.s error which is tabulated at the end as a notified conclusion.

Simulations for the following are carried out to estimate motor speed and position using EKF and UKF.

i. $\quad$ Case-1 Sudden speed change

ii. Case-2 Sudden thrust change

iii. Case-3 Speed reversals

\section{i.Case-1: Sudden speed change}

The motor is set with a speed command of $0.2 \mathrm{~m} / \mathrm{s}$ and at $1 \mathrm{sec}$ a step change in speed command of $0.4 \mathrm{~m} / \mathrm{s}$ is applied. Fig 2(a) to (d) depicts the motor speed and position with and without sensor using EKF and UKF.

\section{(a) Speed Estimation and its error :}

Estimated speed using UKF follows closely the actual speed during transient and steady state. In case of EKF, the estimated speed is deviating from the actual speed during transient state and a constant error is exists during steady state. Whereas error in speed estimation in steady state is almost zero in case of UKF as shown in fig 3(b) 


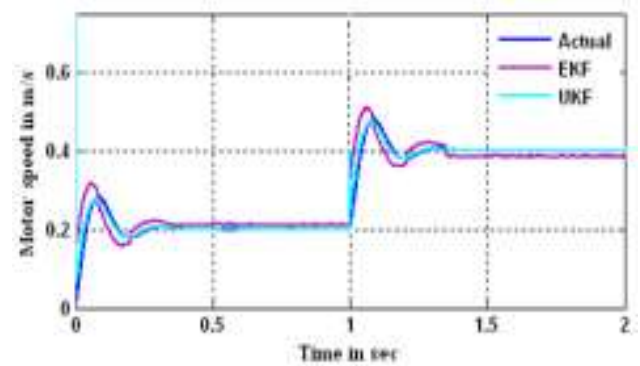

Figure 3(a): Estimated motor speed

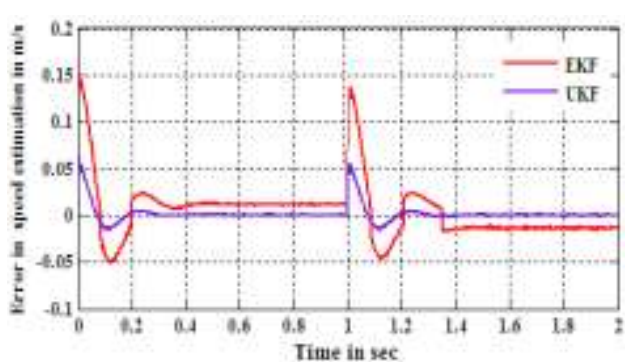

Figure 3(b): Error in estimated speed

(b)Position Estimation and its error: The estimated position obtained from UKF tracks the actual position quite closely whereas estimation using EKF is trailing the actual position. Fig 3(c) \& 3(d) depicts the motor position and the position estimation error in both EKF \& UKF.

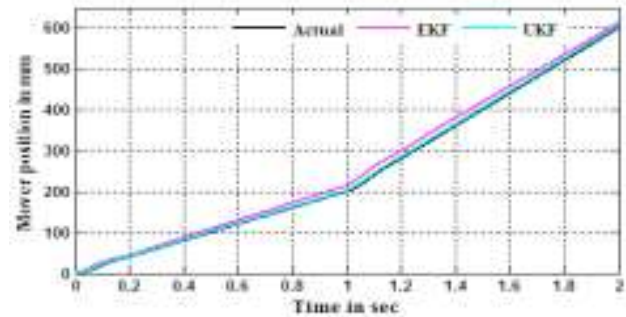

Figure 3(c): Estimated position

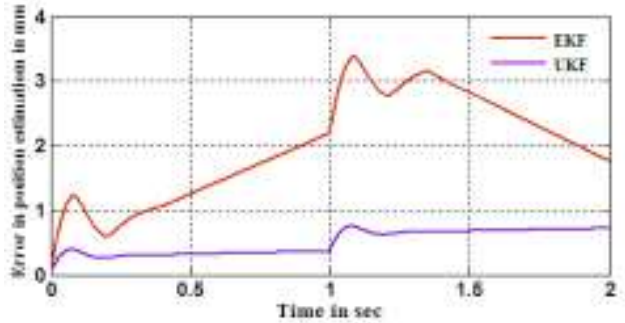

Figure 3(d): Position error

\section{ii. Case-2: Sudden thrust change}

In this case the motor speed is $0.1 \mathrm{~m} / \mathrm{sec}$. and at 1 secsudden load torque of $5 \mathrm{~N}$ is applied, the response is shown in Fig-3(a) to (d).

\section{(a) Speed Estimation:}

It is clear from the fig 4 (a) that the estimated speed using EKF and UKF are within the limit at steady state whereas due to the sudden load applied at $1 \mathrm{sec}$ speed estimation using EKF is deviating from the actual whereas UKF results are quite satisfactory.

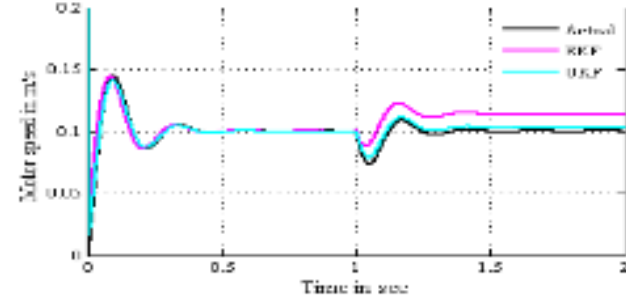

Figure 4(a): Estimated motor speed

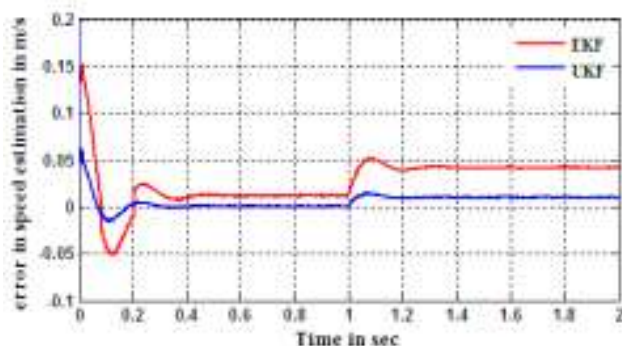

Figure 4(b): Error in estimated speed

(b)Position Estimation : The estimated position obtained from EKF tracks the actual position quite closely upto $1 \mathrm{sec}$. When sudden thrust of $5 \mathrm{~N}$ is applied the estimated position is deviated from the actual as shown in Fig-4(c), Whereas UKF is tracking the actual mover position very closely even when there is a step change in load thrust. For better 
understanding the position is represented in radians. One polepitch dist $(40 \mathrm{~mm})$ is corresponding to $2 \pi$ rad. Position in radian are shown in $4(\mathrm{~d})$.

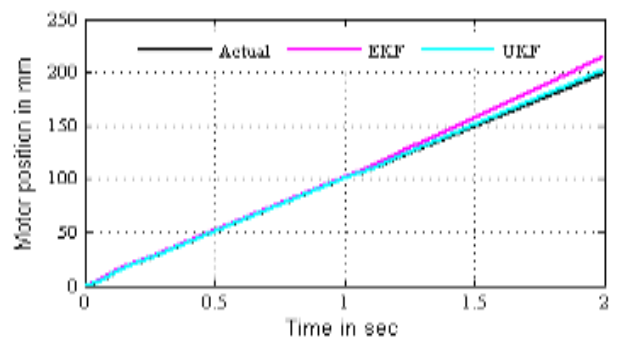

Figure 4(c): Estimated position

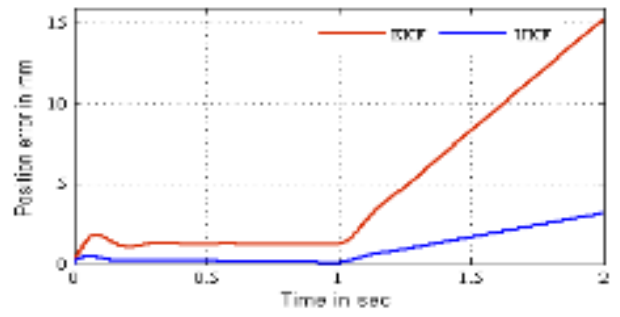

Figure 4(d): Position error

\section{iii. Case-3:Speed reversal}

In this case speed of the motor has reversed from $0.5 \mathrm{~m} / \mathrm{sec}$ to $-0.5 \mathrm{~m} / \mathrm{sec}$.

(a) Estimation of speed : In the case of speed reversal command, the estimated speed using UKF algorithm is extremely near to the actual speed for the entire speed profile. The error in speed estimation by UKF compare to EKF is decreasing $80 \%$ as shown in Fig-5(b).

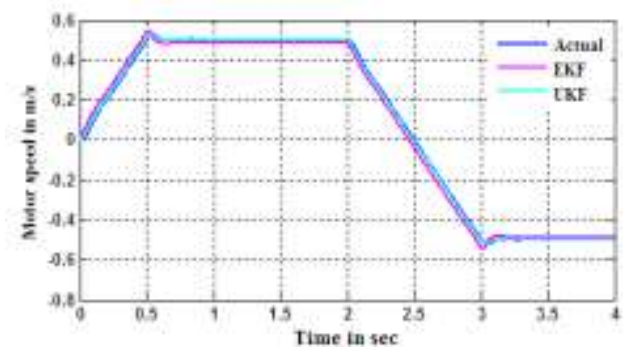

Figure 5(a): Estimated motor speed

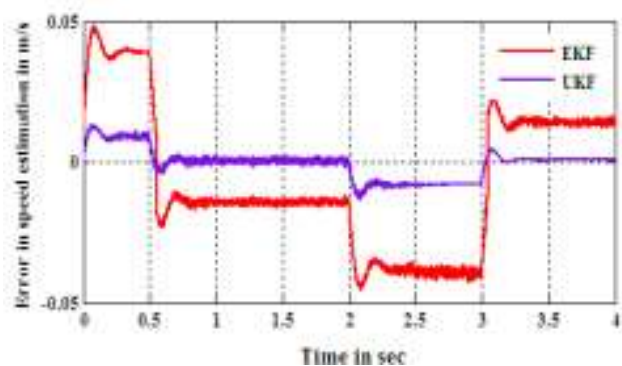

Figure 5(b): Error in estimated speed

(b) Estimation of Mover position: Both the estimators are tracking the actual mover position up to $2 \mathrm{sec}$. During speed reversal UKF is close to actual whereas EKF failing to track the actual mover position. Fig 5(d) gives the clear picture of the position error during speed reversal at $2.5 \mathrm{~s}$.

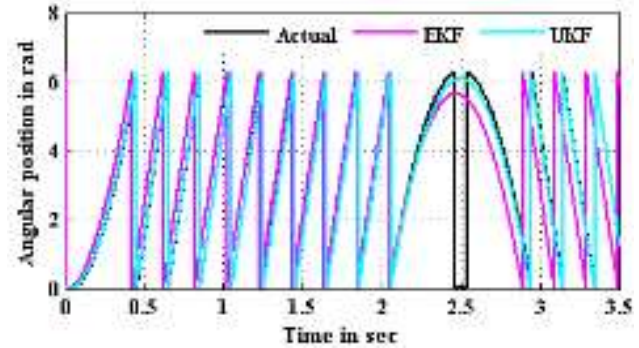

Figure 5(c): Estimated position

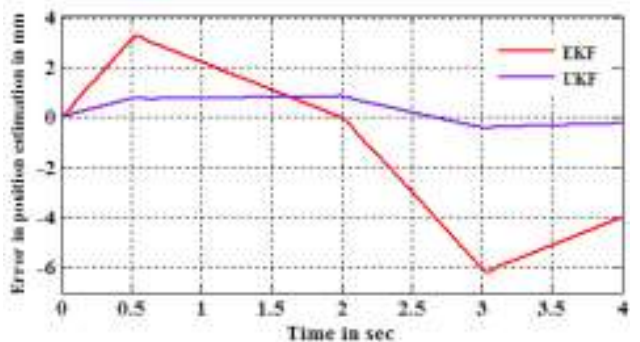

Figure 5(d): Position error 


\section{Performance Comparison}

To compare the performance of both the Kalman estimators, R.M.S error (root mean square error) is calculated considering 500 samples and the estimated errors are shown in Table- 1

$$
\text { R.M.S Error }=\sqrt{\frac{1}{n_{j}} \sum_{j=1}^{n_{j}}\left(x_{j}-\widehat{x}_{j}\right)^{2}}
$$

Where as no. of sample points are denoted as $n_{j}$, the actual state $i s \mathcal{X}_{j}$ and the estimated state is $\widehat{X}_{j}$

\section{Table 1. Comparison of RMS error using EKF and UKF}

\begin{tabular}{|clcccc|}
\hline Sl. & Speed Command & \multicolumn{2}{c}{ Speed error } & \multicolumn{2}{c|}{ Position error } \\
No & & EKF & UKF & EKF & UKF \\
\hline 1 & Case-1 Sudden change in speed & 0.034 & 0.063 & 2.455 & 0.52 \\
& & & & & \\
\hline 2 & Case-2 Sudden change in thrust & 0.042 & 0.0107 & 5.494 & 1.153 \\
\cline { 4 - 5 } & & & & 0.3793 & 0.371 \\
\hline
\end{tabular}

\section{Real Time Implementation}

To implement in real time the complete controller algorithm has programmed using embedded MATLAB and Simulink.DSP code is generated using dSPACE software integrated with MATLAB.DS-1104 control board is used for real time implementation and it provides the DAC and ADC ports and pulse width modulation ports to interface with the sensors and voltage source inverter. The voltage source inverter consists of IGBTs with opto-isolation and gate driver circuit. The DC link voltage and motor phase currents are measured by the LTS-6 Hall-effect sensors. Magnetic sensing type incremental linear encoder with $20 \mu \mathrm{m}$ resolution is used.

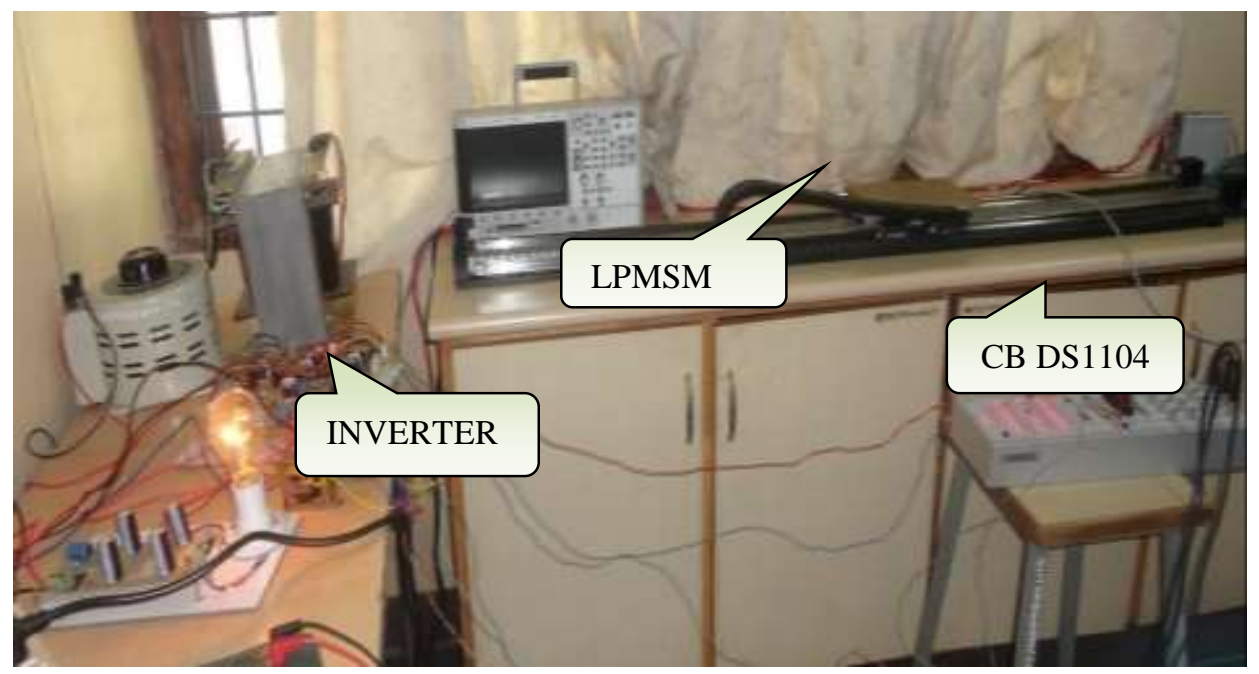

Figure 6. Experimental set-up 


\section{Experimental Results}

Step speed command: For a step change in speed of $0.05 \mathrm{~m} / \mathrm{s}$ at a load thrust of $5 \mathrm{~N}$, the speed and position response with and without encoder (EKF \& UKF) of the motor is shown in Fig-7(a) \& (b)

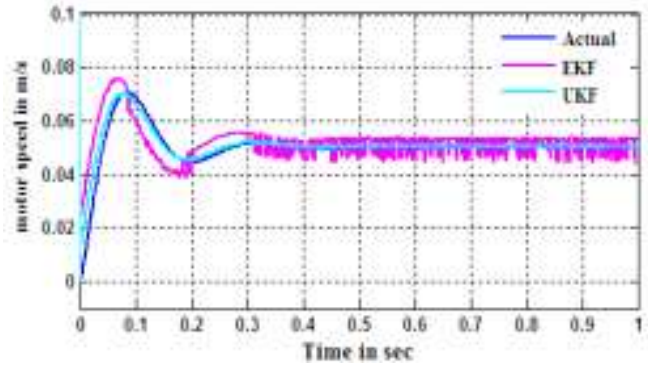

Figure 7(a): Estimated motor speed

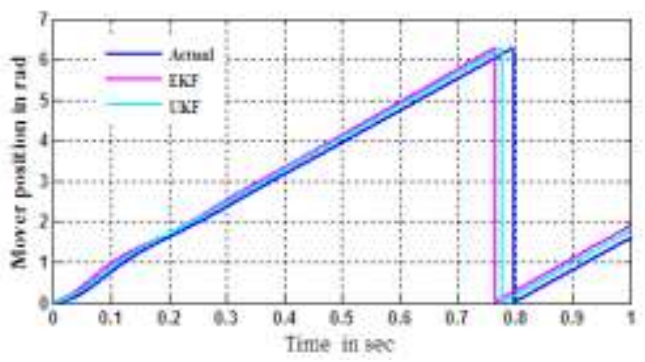

Figure 7(b): Estimated motor Position

It has observed from Figure 7(a) that the estimated speed using UKF is very close to the actual speed. Though at steady state both the estimator performance is similar whereas during transients UKF is estimating quite better than EKF. The estimated speed error for EKF is about $20 \%$ and almost zero in case of UKF.

Experimental results of encoder less control using UKF : The performance of the LPMSM encoder less drive using UKF under various operating conditions are depicted in the Fig. 8 \& Fig. 9 it has observed that the results are satisfactory.

\section{i. Step speed command of $0.1 \mathrm{~m} / \mathrm{s}$ at a constant load thrust of $5 \mathrm{~N}$.}

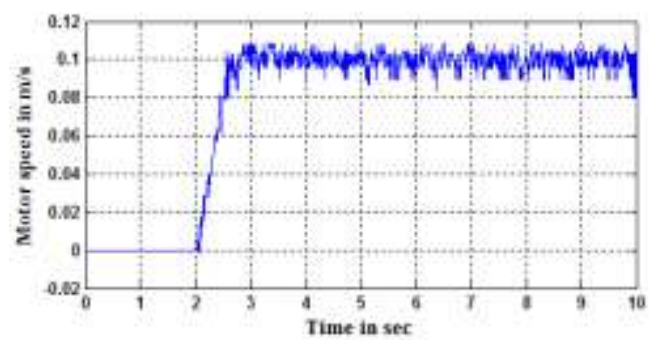

Figure 8(a): Motor speed

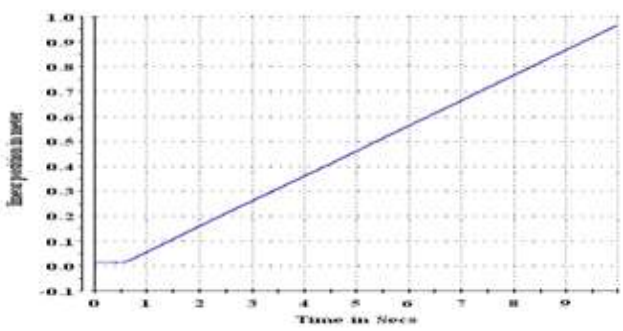

Figure 8(b): Motor Position

ii) Trapezoidal speed command of $0.3 \mathrm{~m} / \mathrm{sec}$ with an acceleration of $0.5 \mathrm{~m} / \mathrm{sec}^{2}$.

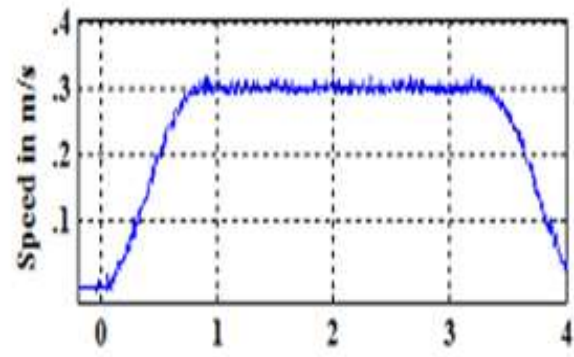

Figure 9(a): Motor speed

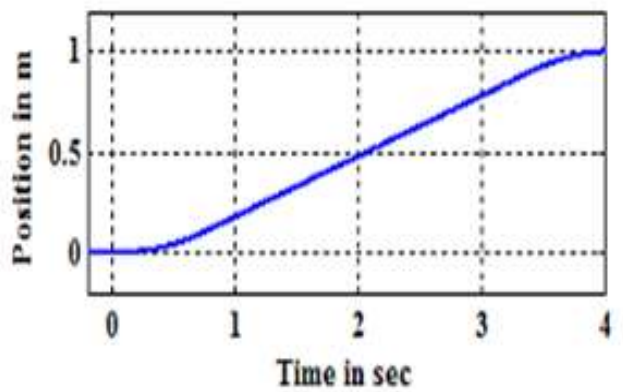

Figure 9(b): Motor Position 


\section{Conclusion}

This paper focus on the estimation of position and speed of a LPMSM drive from the information of motor voltage and current using EKF \& UKF algorithms. A comparative analysis of both the nonlinear estimators has presented with the help of results and its error analysis. Though EKF is estimating correctly in steady state condition, it is not tracking the position and speed correctly during transient conditions like sudden variation in load thrust and speed. Simulation \& Experimental results shows that UKF is performing better during transient as well in steady state conditions. The performance of UKF seems to be more promising with better filtering characteristics compare to EKF. Hence to reduce the cost of the high performance LPMSM drive, UKF is the better choice to replace the encoder.

\section{References}

[1] J. F. Gieras, Z. J. Piech and B. Tomcz. "Linear Synchronous Motors: Transportation and Automation Systems," (Electric Power Engineering series) CRC press, (2000).

[2] W. Yan, H. Lin, and J. Lu, "Sensor-less control of PM Synchronous Motor drive using sliding mode technique", Int. conference Mech. Auto. ICMA, (2009), pp. 3656-3661.

[3] T. Orlowska-Kowalska and M. Dybkowski, "Stator current-based MRAS estimator for wide range speedsensorless induction motor drive,” IEEE Trans. Ind. Electron., vol. 57, no. 4, (2010), pp. 1296-1308.

[4] J. Kang, X. Zeng, Y. Wu. and D.Hu., "Study of position sensorless control of PMSM based on MRAS", IEEE int. conf. Ind. Tech., ICIT, (2009), pp-1-4.

[5] M. Burat, S. Bogosyan and M. Gokasan, "An EKF Based Estimator for Speed Sensorless Vector Control of Induction Motors," Electric Power Components and Systems, vol. 33, no. 7, (2005), pp. 727-744.

[6] G. Rigatos, \& Q.Zhang, "Fuzzy Model Validation using the Local Statistical Approach," Publication Interne IRISA No 1417, Rennes, France.

[7] Simon, Dan, “Optimal State Estimation”, A John Wiley sons, INC., Hoboken, New Jersey. (2006).

[8] VinkoLe`si'c, Mario Va`sak, Goran Stoji ci'c, NedjeljkoPeri’c, GojkoJoksimovi'c and Thomas M. Wolbank, "State and Parameter Estimation for Field-oriented Control of Induction Machine Based on Unscented Kalman Filter," International Symposium on Power Electronics, Electrical Drives, Automation and Motion, (2012), pp. 409-412.

[9] B. Arundhati, K. Alice Mary and M. Suryakalavati, "Implementation of Thrust Ripples Reduction for a Permanent Magnet Linear Synchronous Motor Using Adaptive Feed Forward Controller". Journal of Power Electronics, ISSN 1598-2092, vol. 14, no. 4, (2014) pp.687-694.

[10] S. J. Julier and J. K. Uhlmann, "Unscented Filtering and Nonlinear Estimation," Proceedings of the IEEE, vol. 92, no. 3, (2004), pp. 401-422.

[11] Ravikumar Jagadeesan, Subramanian Srikrishna, Prakash Jagadeesan, "Analysis and Design of Derivative Free Filters against Derivative Based Filter on the Simulated Model of a Three Phase Induction Motor," Energy and Power Engineering, vol. 2, (2010), pp. 78-89.

[12] S. Jafarzadeh, C. Lascu and M. Sami Fadali, "Square Root Unscented Kalman Filters for State Estimation of Induction Motor Drives", IEEE Transactions on Industry Applications, vol. 49, no. 1, (2013), pp.9299 . 